\title{
Smart feeding in farming through IoT in silos
}

\author{
Himanshu Agrawal, Javier Prieto, Carlos Ramos and Juan Manuel Corchado
}

\begin{abstract}
Smart farming practices are of utmost importance for any economy to foster its growth and development and tackle problems like hunger and food insecurity and ensure the well-being of its citizens. However, such practices usually require large investments that are not affordable for SMEs. Such is the case of expensive weighing machines for silos, while the range of possibilities of the Internet of Things (IoT) could intensively reduce these costs while connecting the data to intelligent Cloud services, such as smart feeding systems. The paper presents a novel IoT device and methodology to monitor quantity and quality of grains in silo by estimating the volume of grains at different time instants along with temperature and humidity in the silo. A smart feeding system, implemented via a virtual organization of agents, processes the data and regulates the grain provided to the animals. Experimental on-field measurements at a rabbit farm show the suitability of the proposed system to reduce waste as well as animal diseases and mortality.
\end{abstract}

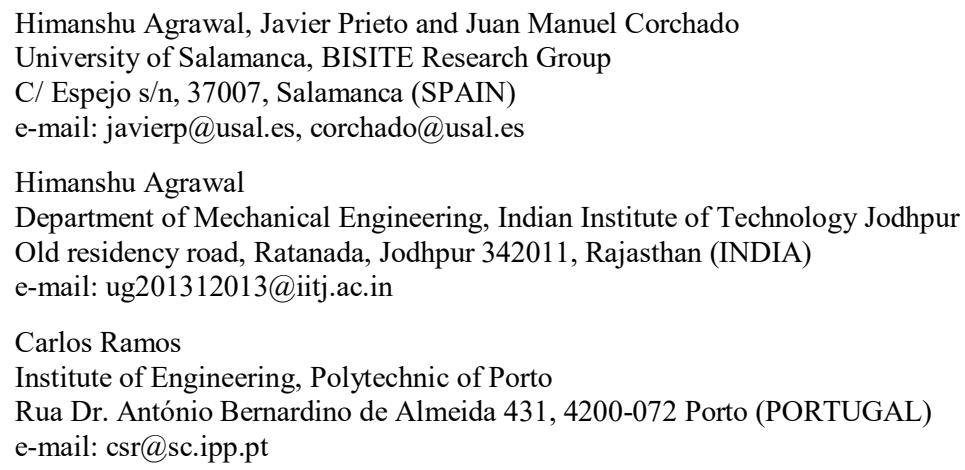




\section{Introduction}

Smart farming techniques have been put in the forefront of many farmers and food produces as long as they can reduce costs, facilitate traceability, and increase security $[1,2,3]$. Many smart farming technologies require large investments in intelligent devices, however, the irruption of the Internet of Things (IoT) is increasingly reducing these investments. Such is the case of expensive weighing machines used to calculate the quantity of grain in silos. Silos are big structures that are used to store bulk quantity of materials like cement, grains, saw dust, food products, etc. (see Fig. 1). In agriculture, they are used to store grains and protect them from being attacked by insects, rodents, birds and also provide a medium to store large quantities of grain over long time with protection. The aim of this paper is to develop an affordable intelligent system able to measure the volume/weight of the grain in different silos, together with ambient conditions, and intelligently use these data to regulate feeding of animals.

But, what is the use of knowing the volume of grains in the silo at different time instants? The information on volume of grains plays a pivotal role in efficient management of stock. By proper monitoring the volume of grains currently present inside the silo, the farmers or industries can do a proper management of their available stock and plan accordingly when they have to refill the stock so that they are not at a risk of out of stock danger. The timely updates on the volume of grains will prevent them from losses incurred due to unavailability of stock and also improve food security and help to fight hunger, where one of the major causes of the hunger is due to improper management of grains after post-harvest. Also the temperature and humidity monitoring of the grains over time can help in ensuring the grain quality and it can alert the farmer of the degradation in the quality of grains, thus not only preventing losses for the farmer but also instrumental in tackling hunger and food insecurity due to poor quality of grains. Moreover, with the farmers and the owners of silo having updates on volume of grains in the silo through the continuous monitoring of the silo content, they are able to plan when they need to purchase the next stock according to the market availability of grains and the price of grains in the market.

In addition, one of the major causes of failure of silos filled with small grain size products is due to the distress in the structure which is caused as sometimes the silo is unable to take the loads of the product which has been filled inside it. Also sometimes failure occur after a little time after the outlet gate has been opened for discharge as during the discharge process the lateral pressure exerted by the stored grains on the walls of the silo exceeds the permissible level, since the pressure exerted by the grains during discharge is more than the pressure exerted by the stored grains and thus resulting in cracks in the walls or total sudden fall down of the structure. But once the user is aware of the volume of the grains inside the silo, the user will be able to prevent situations like overloading of the silo with grains, and prevent keeping silo filled with extra large quantities of grains for a long time and thus it can prevent failure of the silo due to these reasons. 
Some approaches have dealt with the measuring of the volume of grains inside the silo, however, they present different drawbacks such as the requirement of constant contact between grains and sensor [4], or the limitation to liquid materials [5]. Moreover, these systems restrict their functionality to measuring the volume, while additional capabilities such as measuring ambient conditions, and intelligently feeding animals are considered within the virtual organization of agents presented in this paper.

This research paper deals with the development of an IoT device that can measure the volume of the grains inside the silo at different time instants and keep its user timely updated about the volume information along with the temperature and humidity inside the silo so that the user can properly and efficiently manage the quantity and quality of stock and prevent from out of stock risks, thus avoiding losses and providing efficient management. The IoT device sends the data via WiFi connection to a Cloud service that monitors silo and feeds animals. A virtual organization of agents governs the system, where there are intelligent agents in sub-organizations dedicated to collect data, fuse information, control feeding, or active alarms in the case a risky situation is detected $[6,7]$.

The rest of the paper is organized as follows: Section 2 summarizes related work regarding volume measuring at silos, Section 3 states the problem to be solved, Section 4 describes the deployed system, Section 5 discuss the experimental setup and experiments, and, finally, Section 6 draws the conclusions.

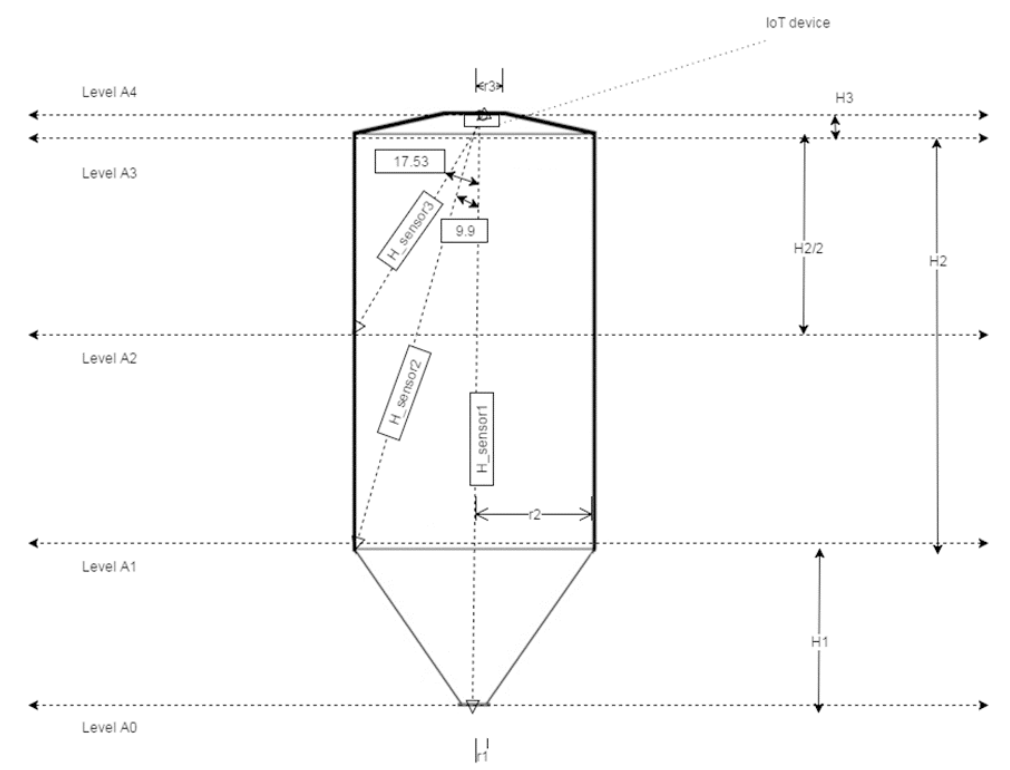

Fig. 1. Silo structure and notations. 
Table 1. Notations

\begin{tabular}{ll}
\hline Notation Meaning \\
\hline $\mathrm{H}_{\text {sensor1 }}$ & $\begin{array}{l}\text { Distance of the obstacle measured from the GH-311 RT Ultrasonic sensor when the } \\
\text { GH-311 RT Ultrasonic sensor mounted on HS-645 MG Servo motor makes an angle } \\
\text { of } 0 \text { degrees with the vertical. }\end{array}$ \\
$\mathrm{H}_{\text {sensor2 }}$ & $\begin{array}{l}\text { Distance of the obstacle measured from the GH-311 RT Ultrasonic sensor when the } \\
\text { GH-311 RT Ultrasonic sensor mounted on HS-645 MG Servo motor makes an angle } \\
\text { of } 9.9 \text { degrees with the vertical. }\end{array}$ \\
$\mathrm{H}_{\text {sensor3 }}$ & $\begin{array}{l}\text { Distance of the obstacle measured from the GH-311 RT Ultrasonic sensor when the } \\
\text { GH-311 RT Ultrasonic sensor mounted on HS-645 MG Servo motor makes an angle } \\
\text { of } 17.53 \text { degrees with the vertical. }\end{array}$ \\
$\alpha_{2}$ & $\begin{array}{l}9.9^{\circ}=\text { angle made by the GH-311 RT Ultrasonic sensor mounted on the rotating HS- } \\
645 \text { MG Servo motor with the vertical }\end{array}$ \\
$\alpha_{3}$ & $\begin{array}{l}17.53^{\circ}=\text { angle made by the GH-311 RT Ultrasonic sensor mounted on the rotating HS- } \\
645 \text { MG Servo motor with the vertical }\end{array}$ \\
$\mathrm{r}_{1}$ & $\begin{array}{l}\text { Radius at level AO } \\
\mathrm{r}_{2}\end{array}$ \\
$\mathrm{r}_{3}$ & $\begin{array}{l}\text { Radius at level A1 } \\
\mathrm{H}_{1}\end{array}$ \\
$\mathrm{H}_{2}$ & $\begin{array}{l}\text { Height from level A0 to A1 level A4 } \\
\mathrm{H}_{3}\end{array}$
\end{tabular}

\section{Related work}

The problem of estimating the approximate volume of the grains in the silo for quantity monitoring along with temperature and humidity measurement for quality monitoring has existed from a long time. This problem not only plays a vital role for farmers and industries but also is an important aspect of ensuring food security. Work related to this problem background of quantity monitoring inside silo has been done in the past in the form of level measurement techniques of the fluid present in the silo where this fluid could be solid like grains or liquid by making use of appropriate sensor.

Grain level measurement technique by making use of capacitance based sensors has been deployed in [4] to measure the grain level inside the silo. Their sensor simulation showed that the readings were in accordance with the theory with the condition being that the reference sensor was filled with grain completely (whereas by making use of ultrasonic here we can make non-contact measurements of the silo content). The limitation mentioned by them is that if one needs to know the grain content inside the silo by using the grain level measurement readings then it can come out to be faulty and this error is difficult to avoid for level measuring systems for solids like grain, powders, etc. as the grain accumulation inside the silo is not flat and it possesses a surface profile. This motivated a re- 
search to be carried out in the volume estimation of the grain content inside the silo.

A sensor based fluid level measurement system has been applied in [5] where the sensor design is based on a passive circuit comprising of inductor and capacitor and the substance should be able to be placed inside the capacitance plates. In a study on "liquid level sensor using ultrasonic lamb waves" [8], ultrasonic lamb waves are used to detect the presence of liquid as the wave characteristic changes when it comes in liquid contact. Liquid level measurement by using and applying technology of optical fibers has been applied in [9] which makes use of the measurement in the variations in amplitude where it is a function of the distance of the liquid.

Work related to the problem background of quality monitoring has been done through studying the temperature variations and moisture variations in the grains inside the silo by installing the sensors at different points inside the silo. The temperature variations in the headspace are larger than the temperature variations inside the grain mass and also the moisture change inside the grain mass is lower than the moisture change in the surface grain [10]. Also, when a large dry bulk of material is exposed at the surface to high relative humidity then the moisture diffusion rate through the material is extremely slow in practical conditions [11]. This motivated us to install the temperature and humidity sensor at the headspace in the silo rather than installing it at different locations inside the silo for temperature and humidity monitoring.

The quality monitoring plays an important role in detecting the presence of beetles in the grains inside the silo. In a study on the influence of relative humidity and thermal acclimation on the survival of adult grain beetles in cooled grain [12] it was found that the survival rate was shorter at $45 \%$ relative humidity than at $70 \%$ relative humidity. Also it was found that at $45 \%$ relative humidity all species survived when the grain temperature was at $13.5^{\circ} \mathrm{C}$ but when the temperature was further brought down to 9 degrees, only S. granaries did survive 26 weeks. Also, in a study on the effects of temperature on the aerobic stability of wheat and corn silages [13], it was found that silage kept at $30^{\circ} \mathrm{C}$ had the highest production of $\mathrm{CO}_{2}$, largest $\mathrm{pH}$ increase and the largest count of yeast than the samples kept at 10,20 , and $40^{\circ} \mathrm{C}$. The samples kept at 10 and $40^{\circ} \mathrm{C}$ were found to be stable. So this motivated to measure temperature and humidity inside the silo to monitor grains over time.

\section{Problem statement}

Since the level of grains inside the vertical silo is not known and also the surface profile of the top portion of the grains inside the vertical silo is not known, it makes it difficult to estimate the quantity or volume of grains inside the silo at different time instants. 
Without an effective system to measure the quantity or volume of grains inside the silo at different time instants, it hinders proper and efficient management of stock of grains thus, sometimes leading to scarcity of grains to the people leading to hunger and food insecurity and sometimes there are excess grains leading to losses to the farmers and industries and wastage of grains.

Without proper quality monitoring of grains in silo, the degradation in quality of grains could not be managed and thus it can lead to grain shortage, hunger, food insecurity and loss to the farmers.

In the following, we develop an IoT device that can give timely updates of the volume of grains, $V_{t, t \in \mathrm{N}}$, inside the silo at different time instants, $t \in \mathrm{N}$, along with the temperature, $T_{t, t \in \mathrm{N}}$, and humidity, $M_{t, t \in \mathrm{N}}$, data to monitor the quality of grain inside the silo. The IoT device shall be located at the top inside the silo and include an RF module that transmit data to an intelligent Cloud system, based on a virtual organization of agents, which decides whether to increase or reduce feeding of animals taking into account: consumed grain, temperature, humidity, animal age, animal weight, weight evolution, and the probability of disease.

Assumption: The surface profile of the top portion of the grains present in the silo is assumed conical.

There can be different cases depending at what level the grains are present. The different cases can be (see Fig. 2):

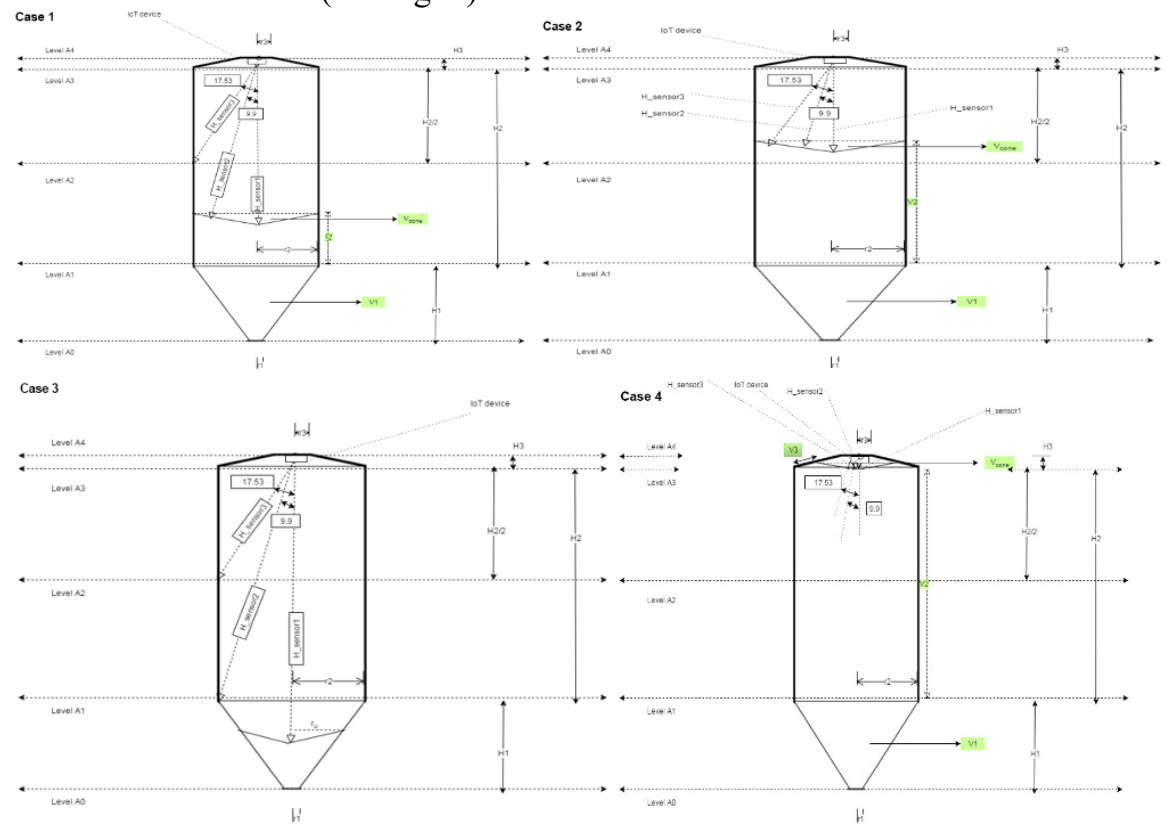

Fig. 2. Cases 1, 2, 3 and 4, respectively, can be distinguished depending on the grain level. 
- Case 1: when the grain level is between A1 and A2. If this case holds, then, $H_{3}+H_{2} / 2<H_{\text {sensor } 1}<H_{3}+H_{2}$, and $V_{t}=V_{1}+V_{2}-V_{\text {cone }}$.

- Case 2: when the grain level is between A2 and A3. If this case holds, then, $H_{3}<H_{\text {sensor } 1}<H_{3}+H_{2} / 2$, and $V_{t}=V_{1}+V_{2}-V_{\text {cone }}$.

- Case 3: when the grain level is below A1. If this case holds, then, $H_{3}+H_{2}<H_{\text {sensor } 1}<H_{3}+H_{2}+H_{1}$, and calculation of $V_{t}$ is straightforward.

- Case 4: when the grain level is above A3. If this case holds, then, $0<H_{\text {sensor } 1}<H_{3}$, and $V_{t}=V_{1}+V_{2}+V_{3}-V_{\text {cone }}$.

$H_{\text {sensor } 1}, H_{\text {sensor } 2}$ and $H_{\text {sensor } 3}$ are the distances measured at the positions marked in Fig. 1, which correspond with angles 0, 9.9 and 17.53 degrees with the vertical, respectively. These angles were calculated based on the geometry of the silo. Then, the grain volume can be obtained, respectively, for the different cases once the values of $H_{\text {sensor } 1}, H_{\text {sensor } 2}$, and $H_{\text {sensor } 3}$ are known.

\section{System description}

The IoT device contains an ultrasonic sensor mounted on a servo motor that rotates to measure the three distances marked in Fig. 1 between the top of the silo and the grain level. It also includes a temperature and humidity sensor that informs the user about temperature and humidity measurement data along with the volume of grains inside the silo at different time instants. The results are shown on a website and an Android Smartphone application. This information can help to eradicate the problems like scarcity of grains and wastage of grains caused due to the presence of unknown amount of grains or poor quality of grains inside the silo and thus help in efficient quantity and quality management of stock. Fig. 3 depicts the different modules that constitute the IoT device. It comprises:

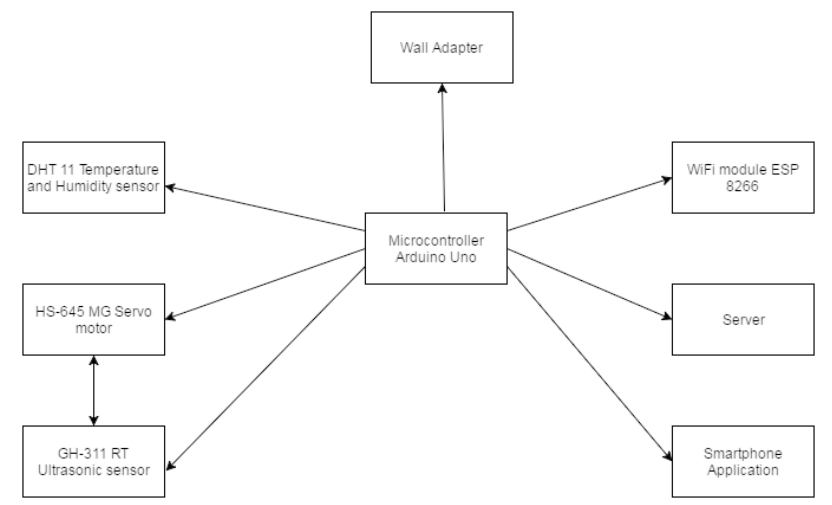

Fig. 3. Arduino Uno Microcontroller enables handling the different blocks that constitute the designed IoT device. 
1. Arduino Uno: It is a microcontroller board based on ATmega328P that controls all other modules.

2. GH-311 RT ultrasonic sensor: This ultrasonic sensor is used in the device to measure the distance between the top of the silo and the grains at different angles. The sensing range of the sensor is $2-8000 \mathrm{~mm}$.

3. HS-645MG Servo motor: The GH-311 RT ultrasonic sensor is mounted on the HS-645MG Servo motor so as to take the distance readings of the obstacle at 3 different angles namely $0,9.9$ and 17.53 degrees. The servo motor operates at a speed of $0.24 \mathrm{~s} / 60^{\circ}$.

4. DHT 11 Temperature and Humidity sensor: The DHT 11 sensor is used to measure the temperature and humidity inside the silo at different time instants. The DHT 11 sensor has a measurement range of $20-90 \% \mathrm{RH}$ and $0-50{ }^{\circ} \mathrm{C}$. The humidity accuracy is $\pm 5 \% \mathrm{RH}$ and the temperature accuracy is $\pm 2{ }^{\circ} \mathrm{C}$.

5. WiFi Module - ESP8266: The WiFi module is used in the IoT device to connect the microcontroller to a WiFi network and helps in transferring the data received from the $3 \mathrm{GH}-311 \mathrm{RT}$ sensor to the server via WiFi.

6. Wall Adapter: The wall adapter is used to convert the AC voltage from the power supply to the DC 5 volt supplied to the Arduino Uno.

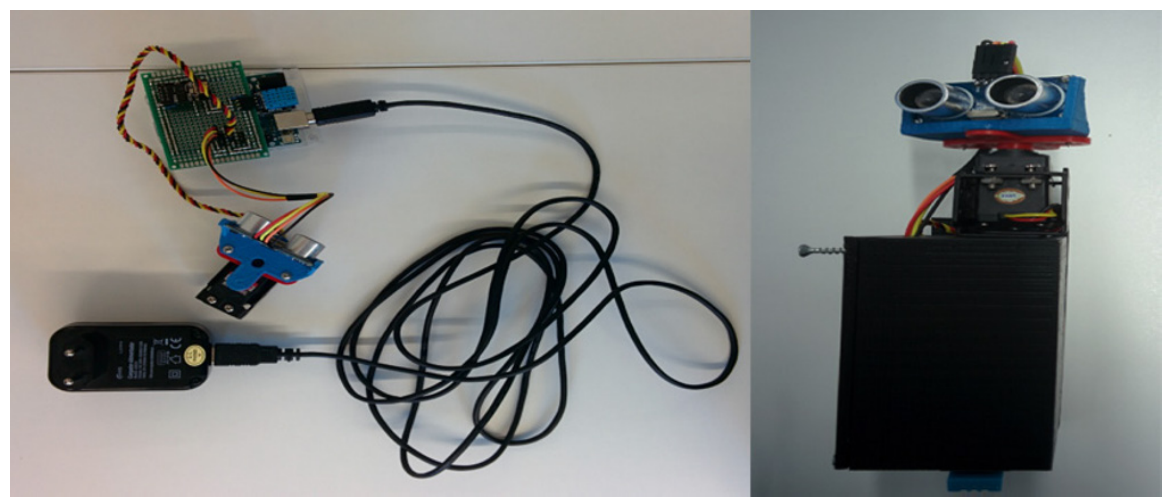

Fig. 4. Prototype of IoT device.

A server application calculates the volume of grains inside the silo at different time instants by making use of the data obtained from the GH-311 RT Ultrasonic sensor mounted on the rotating HS-645MG Servo motor. This quantity is translated into kilograms of grain based on the density of the filled variety. Finally, this value is used to decide whether to increase or decrease feeding.

The server application is based on a virtual organization of agents. Virtual organizations can be considered an evolution of multi-agent architectures, which have been extensively explored as an alternative to develop context-aware systems [14]. This new perspective offers organizational norms, roles and the services associated with them. In the proposed system, there coexist different organizations that jointly solve the smart feeding problem based on the established rules from case-based reasoning on past experiences [15] (e.g., it is known that low or too 
high feeding can derive in animal death): data gathering, context, rules establishment, decision making and feeding regulation organizations. The abovementioned variables influence the feeding rate: consumed grain, temperature, humidity, animal age, animal weight, weight evolution, and the probability of disease

Finally, the server application displays data (volume, temperature, humidity, etc.) inside the silo at different time instants on the web, and a Smartphone application fetches the data from the server and displays it to the user.

\section{Experimental evaluation}

In this section, we evaluate the case study of the smart feeding system working on a rabbit farm. We first describe the experiment and discuss the results afterwards.

\subsection{Experimental setup}

The IoT device was fitted at the lid of the silo at the top such that the IoT device faces the grains. The silo was the model Aviporc 230/5, with diameter $2.3 \mathrm{~m}$, height 8.15 and capacity $21.07 \mathrm{~m}^{3}$ (approximately 12 tons of grain). The device was tested during 10 hours where the silo was filled with 6 tons of corn (approx. $\left.10,5 \mathrm{~m}^{3}\right)$, and 1 ton was pulled out at intervals of 100 kilograms every hour.

The values of $H_{\text {sensor } 1}, H_{\text {sensor } 2}$, and $H_{\text {sensor } 3}$ were determined with the help of the GH-311 RT Ultrasonic sensor mounted on the HS-645MG Servo motor, both of them were connected to the Arduino Uno. The HS-645MG Servo motor rotated from 0 to 25 degrees and the distance readings from the GH-311 RT Ultrasonic sensor were recorded when the GH-311 RT Ultrasonic sensor mounted on the HS-645MG Servo motor made angles of 0, 9.9 degrees and 17.53 degrees from the vertical of the silo.

According to measured $H_{\text {sensor } 1}, H_{\text {sensor } 2}$, and $H_{\text {sensor } 3}$ values, the appropriate case can be determined and it will tell us where the grains are present at a time instant. With this information, the volume of the grains in the silo at different time instants can be known by using the geometry of the given silo.

The DHT 11 Temperature and Humidity sensor was also connected to the Arduino Uno. The DHT 11 sensor calculates the temperature and humidity inside the silo every 5 minutes.

The server application connects to the MySQL database and stores all the information on sensor's values like: Temperature, Humidity, $H_{\text {sensor } 1}, H_{\text {sensor } 2}$, $H_{\text {sensor } 3}$ and volume of the grains in the silo in the database.

Finally, the Smartphone application connects to the server and fetches the data from the server's database about the volume of the grains present in the silo at any 
instant of time along with the values of temperature and humidity. The Smartphone application displays this data to the user.

\subsection{Experimental results}

Fig. 5 shows the actual and measured values of grain during the mentioned 10hours interval, together with measured temperature and humidity readings.

a).

Weight of grains inside the silo (in $\mathrm{kg}$ )

6,500

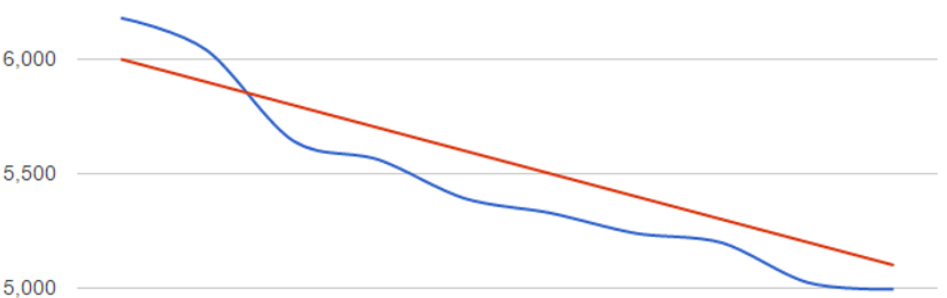

4,500

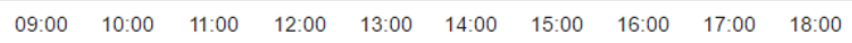

— Measured value - Actual value

b).

Temperature in the silo (in Celcius)

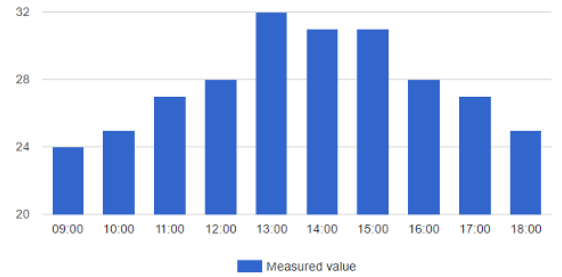

c). Humidity in the silo (in \%)

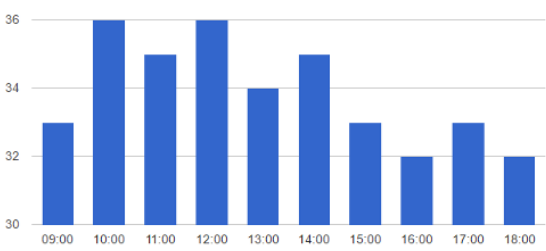

Fig. 5. The developed device smoothly estimates a) the volume of grain; b) the temperature; and c) the humidity in the silo, without the need of large investments for weighing machines.

The figure reflects the suitability of the proposed system where the error in weight estimation is below $4 \%$ in all the considered cases, thus, enabling the smart feeding system to reduce waste and animal mortality. 


\section{Conclusions}

This paper has presented a novel approach for monitoring the quantity and quality of grains inside a silo as this plays a pivotal role in the field of agriculture and ensuring food security, thus tackling hunger and providing efficient and effective management of grain stock along with management of quality of grains. We have developed a novel IoT device based on ultrasonic sensors that measures the grain volume as well as ambient conditions, and sends this data to a Cloud application, based on a virtual organization of agents, that regulates feeding based on these data and case-based reasoning. The experimental results in a rabbit farm show that the proposed solution can be a very beneficial tool for the farmers, industry, individuals who own silo, etc. and help them in properly optimizing the available resources and in taking well informed decisions.

Acknowledgments This work has been partially supported by the European Commission (Seventh Framework Programme for Research and Technological Development) FP7-PEOPLE-2012IRSES project EKRUCAmI (Europe-Korea Research on Ubiquitous Computing and Ambient Intelligence) under grant Ref. 318878.

\section{References}

1. Stein, A.; Allard, D.; van de Kerkhof, B.; van Persie, M.; Noorbergen, H.; Schouten, L.; Ghauharali, R. (2015). Spatio-temporal Analysis of Remote Sensing and Field Measurements for Smart Farming, Procedia Environmental Sciences, 27:21-25.

2. Ryu, M.; Yun, J.; Miao, T.; Ahn, I.Y.; Choi S.C.; Kim, J. (2015). Design and implementation of a connected farm for smart farming system, IEEE Sensors 2015.

3. Andrewartha S.J.; Elliott N.G.; McCulloch J.W.; Frappell P.B. (2015). Aquaculture Sentinels: Smart-farming with Biosensor Equipped Stock, Journal of Aquaculture Research \& Development 7(1):1-4.

4. İşiker, H.; Canbolat, H. (2009). Concept for a novel grain level measurement method in silos. Computers and electronics in agriculture 65(2):258-267.

5. Woodard, S.E.; Taylor, B.D. (2007). A wireless fluid-level measurement technique. Sensors and Actuators A: Physical 137(2):268-278.

6. Villarrubia, G., De Paz, J. F., Bajo, J., \& Corchado, J. M. (2014). Ambient agents: embedded agents for remote control and monitoring using the PANGEA platform. Sensors, 14(8):13955-13979.

7. Tapia, D. I., Fraile, J. A., Rodríguez, S., Alonso, R. S., \& Corchado, J. M. (2013). Integrating hardware agents into an enhanced multi-agent architecture for Ambient Intelligence systems. Information Sciences, 222:47-65. 
8. Sakharov, V. E., Kuznetsov, S. A., Zaitsev, B. D., Kuznetsova, I. E., \& Joshi, S. G. (2003). Liquid level sensor using ultrasonic Lamb waves. Ultrasonics, 41(4):319-322.

9. Vázquez, C., Gonzalo, A. B., Vargas, S., \& Montalvo, J. (2004). Multi-sensor system using plastic optical fibers for intrinsically safe level measurements. Sensors and Actuators A: Physical, 116(1):22-32.

10.Jian, F., Jayas, D. S., \& White, N. D. (2009). Temperature fluctuations and moisture migration in wheat stored for 15 months in a metal silo in Canada. Journal of stored products research, 45(2):82-90.

11.Pixton, S. W., \& Griffiths, H. J. (1971). Diffusion of moisture through grain. Journal of Stored Products Research, 7(3):133-152.

12.Evans, D. E. (1983). The influence of relative humidity and thermal acclimation on the survival of adult grain beetles in cooled grain. Journal of Stored Products Research, 19(4):173-180.

13.Ashbell, G., Weinberg, Z. G., Hen, Y., \& Filya, I. (2002). The effects of temperature on the aerobic stability of wheat and corn silages. Journal of Industrial Microbiology and Biotechnology, 28(5):261-263.

14.Villarrubia, G., De Paz, J. F., Bajo, J., \& Corchado, J. M. (2014). Ambient agents: embedded agents for remote control and monitoring using the PANGEA platform. Sensors, 14(8):13955-13979.

15.Corchado, J. M., \& Laza, R. (2003). Constructing deliberative agents with case-based reasoning technology. International Journal of Intelligent Systems, 18(12):1227-1241. 